\title{
La construcción de valores intangibles de marcas de cerveza artesanal. Atributos inmersos en las comunicaciones
}

\section{The construction of intangible values in craft beer brands. Attributes immersed in communications}

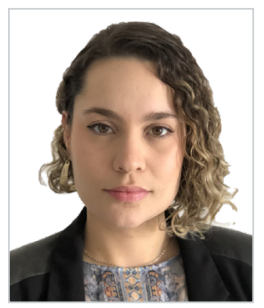

Catalina Osorio Parra. Psicóloga. Master en comportamiento del consumidor de la Universidad Pontificia Bolivariana. Investigadora/becaria del grupo Epilión de Publicidad de la Universidad Pontificia Bolivariana por 2 años. Conferencista en temas relacionados con investigación profunda del consumidor desde una aproximación cualitativa y con foco en las ciencias sociales y humanas especialmente la psicología, el psicoanálisis, sociología antropología y semiótica. Autora de capítulos de libros académicos, artículos especializados para congresos internacionales (Barcelona). Ha trabajado como investigadora Senior en firmas de consultoría de mercados y actualmente trabaja en Sura Seguros Colombia investigando, diseñando e innovando en nuevas propuestas de valor para la organización.

Universidad Pontificia Bolivariana, Colombia

catalina.osoriopa@upb.edu.co

ORCID: 0000-0002-6358-8847

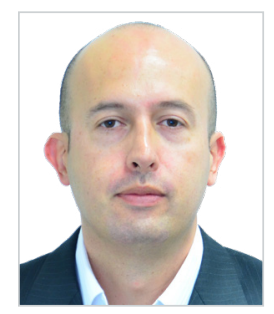

Jaime Alberto Orozco-Toro. Doctor en Medios, Comunicación y Cultura de la Universidad Autónoma de Barcelona (España). Magíster en Publicidad y Relaciones Públicas (UAB). Magíster en Desarrollo de la Universidad Pontificia Bolivariana (Colombia). Conferencista en temas relacionados con publicidad, responsabilidad social corporativa, marca y reputación corporativa en América y Europa. Autor de capítulos de libros académicos y artículos científicos en revistas de Argentina, Chile, Colombia, España, Inglaterra y México. Autor de seis libros sobre publicidad, relaciones públicas, marca y estrategia. Ganador del premio extraordinario a la mejor tesis doctoral de la Universidad Autónoma de Barcelona (España). Integrante del grupo de investigación Epilión (UPB, Colombia), y Comress (UAB, España).

Universidad Pontificia Bolivariana, Colombia

jaime.orozco@upb.edu.co

ORCID: 0000-0002-8152-7474

Recibido: 14/01/2020 - Aceptado: 31/03/2020

Received: 14/01/2020 - Accepted: 31/03/2020

\section{Resumen:}

Este estudio pretende analizar, desde un enfoque hermenéuticoetnográfico, la construcción de valores intangibles del consumidor de las marcas de cerveza artesanal producidas en MedellínColombia, en relación a su estrategia de comunicación. Las bases

\section{Abstract:}

This study took a hermeneutic-ethnographic approach and aims to analyse the construction of intangible values in consumers of craft beer brands produced in Medellin-Colombia and its relation to their communication strategy. The theoretical bases are built

Cómo citar este artículo:

Osorio Parra, C.; Orozco-Toro, J. A. (2020). La construcción de valores intangibles de marcas de cerveza artesanal. Atributos inmersos en las comunicaciones. Doxa Comunicación, 30, pp. 249-263. 
teóricas se fundamentan desde los conceptos de identidad, imagen y valores intangibles de marca. Dentro de los resultados más importantes se puede destacar que la cerveza artesanal responde a un nuevo segmento como resultado de nuevas estrategias comunicativas e imaginarios alrededor de lo artesanal. Además, los valores intangibles más relevantes que perciben los usuarios de las marcas giran alrededor de la confianza, la innovación, el orgullo, entre otros. Mediante esta investigación se demuestra la importancia que tienen los valores intangibles, especialmente en la comunicación y en la construcción de una correcta imagen de marca.

Palabras clave:

Valores intangibles; identidad; imagen; cerveza artesanal; estrategia comunicativa; microcervecerías; imaginarios; construcción de marca.

\section{Introducción}

La investigación de los Valores Intangibles (V.I.) ha sido un tema relevante para los campos de la comunicación y el marketing por su importancia en la construcción de imagen e identidad corporativa de las marcas. Además, porque de esta manera se puede entender con mayor claridad el proceso de evaluación, que sobre los V.I., realizan los consumidores. Basado en estas circunstancias, este estudio realiza una indagación sobre la construcción de los valores intangibles en marcas de cerveza artesanal, con lo cual se pretende aportar nuevas miradas a un tema que por su novedad y por su escasa literatura reviste un significativo interés, no solo desde la visión empresarial, sino desde las actitudes de los consumidores. En Colombia, no se ha realizado un análisis de las estrategias comunicativas de las cervezas artesanales y mucho menos desde las representaciones sociales y las construcciones culturales de los V.I. que emite la marca y que son percibidos por los consumidores. Para comprender el proceso de generación de estos por parte de las marcas, y la forma en que los consumidores los perciben, se requiere comprender los conceptos asociados, como identidad, imagen y estrategia comunicativa.

\subsection{Identidad de marca}

La identidad de marca ha sido definida como aquello que es comunicado por medio de una multiplicidad de signos, mensajes y productos, emanado de un único emisor (Kapferer, 1992; Elizondo, Bouchard \& Favart, 2016; Apolo, Báez, Pauker \& Pasquel, 2017). No obstante, algunos autores sostienen que la identidad es mucho más que eso, asegurando que " $a$ brand is also far more than the name, logo, symbol or trademark that highlights its origin; it is imbued with a set of unique values that defines its character" (Slade, 2016: 12), resaltando la importancia de tener claridad de aquellos valores que la vuelven única. De acuerdo con lo anterior Orozco-Toro (2018) define la identidad como la materialización de los aspectos que identifican a la empresa en atributos dignos de ser comunicados a los stakeholders. Para Semprini (1995) la identidad es la 
forma en la que una marca se hace visible y se materializa en los discursos que los actores sociales cruzan entre sí, dando importancia a la construcción del perceptor del mensaje respecto a estos atributos. Otros estudios van aún más allá y resaltan que la identidad de marca, desde una posición estratégica, es la representación de la empresa y sus características únicas y distintivas logradas desde el producto, los signos, símbolos, diseños o estrategias de diferenciación (Buil, Catalán \& Martínez, 2016). Incluso se describe la identidad como una barrera, ya que "Corporate brands can block the trespass of a rival with their distinctiveness" (Gehani, 2016: 11). La generación de una correcta identidad de marca será la que le permita a la empresa una correcta construcción de la imagen ante sus diferentes stakeholders. Así lo entiende Sanz de la Tajada (1996) al destacar que para conseguir una imagen controlada la empresa debe proyectar su propia personalidad e identidad. Es por esto que la estrategia comunicativa y la identidad cobran importancia pues serán responsables de condicionar la imagen de marca. En este caso Slade (2016) establece la importancia de la identidad en términos de generación de valores intangibles que permitan una representación de la imagen de la marca en la mente de los stakeholders. Finalmente, y para reafirmar esta condición de vínculo entre la identidad y la imagen, se puede advertir que: "Marketers are trying, in effect, to "paint a picture" of their brands in the minds and hearts of consumers" (Keller, 2016: 291).

\subsection{Imagen de marca}

La imagen de marca es el resultado del amplio proceso, que se inicia con el comportamiento de la compañía y la gestión estratégica y comunicativa de su identidad, y que finaliza con la generación de actitudes por parte de los stakeholders (Millares-Marcelo, Millares-Quirós \& Daza-Izquierdo, 2014). La imagen de marca es un proceso complejo, pues representa "the successful building of a brand image is never accidental" (Chao-Sen, 2015: 234). Según este autor se puede tener una buena marca, una correcta identidad, un producto con gran funcionalidad, pero estos factores deben redundar en una buena imagen de marca pues es allí donde se reafirma la satisfacción de los consumidores. Por ello, la imagen de marca es un concepto de recepción, complejo y multidimensional, producto y resultado de las construcciones mentales de los stakeholders (Gazquez \& Sánchez, 2004), y que además consiste en percepciones y las asociaciones que los grupos de interés conservan en su memoria (Keller, 2008).

Algunos autores la definen como la representación mental del consumidor respecto a la oferta donde significados construidos desde lo simbólico están asociados con las características específicas de un producto o servicio (Manhas, Manrai \& Manrai, 2016; Turan, 2017), resaltando una imagen clara con el objetivo de satisfacer exitosamente las necesidades del consumidor. Por su parte, Che-Hui, Miin-Jye, Li-Ching \& Kuo-Lung (2015) afirman que estas asociaciones mentales respecto a las marcas tienen unos niveles de intensidad, donde la conexión con una marca está basada en la experiencia del consumidor y el resultado de su exposición a las comunicaciones. Hoy en día se reconoce que estas asociaciones se pueden entender desde los atributos, los beneficios y las actitudes hacia la marca. En este sentido, Finne \& Grönroos (2009) expresan que las marcas han tenido que mudar su foco de atención, dejándose de basar en el paradigma en donde la compañía es la que produce la integración de un mensaje:

Instead of the traditional marketing communication concept, where a sender conveys a message to a receiver through coding, noise and decoding processes, marketing communication should focus more on the receivers and the meaning created by the receiver in the communication process (Finne \& Grönroos, 2009: 180). 
Lo anterior evidencia la necesidad de las marcas de enfocarse en el consumidor, pues es el encargado de cumplir con la labor de integración de los mensajes, acentuando la importancia de la creación de significados, construidos a partir de esos atributos anteriormente mencionados, conceptualmente hoy nombrados como los valores intangibles de una marca, que son dados a conocer en las estrategias comunicativas de la organización.

\subsection{Valores intangibles de marca}

Los conceptos de identidad e imagen de marca son esenciales para entender la teoría de los valores intangibles. La primera representa la estrategia comunicativa que la compañía quiere transmitir y la segunda la construcción que los stakeholders edifican de la organización. Dentro del mensaje entre marca y consumidor entran en juego una serie de atributos llamados valores intangibles, que se construyen y gestionan desde la estrategia de marketing. En el momento en que los stakeholders, y en especial los consumidores, comienzan a interpretarlos es cuando se genera la construcción de los mismos. Por ejemplo, autores como Pizzolante (2009), consideran que la calidad de los productos ya no es el único parámetro competitivo de una marca, por lo que la intangibilidad a través de los V.I. se convierte en una parte primordial de cómo los consumidores perciben las marcas. Reforzando lo anterior: "brand value as a corporate asset is one of the primary measures of competitive advantage of an enterprise that is useful to gain customers' brand preference over rivals" (Gehani, 2016: 11).

La preocupación por evaluar la intangibilidad de las marcas y sus V.I., parte de la necesidad de desarrollar relaciones duraderas con el fin de crear y mantener el valor del cliente, pensando más allá de las meras transacciones y la venta de un producto (Porcu, Del Barrio-García \& Kitchen, 2012). Entender el comportamiento de los consumidores y cómo construyen los valores intangibles es pensar en la sostenibilidad y el futuro de las marcas, por lo tanto de las empresas, y de la generación de valor que de esta relación provoca. Desde la perspectiva de la marca se debe considerar que la lista de V.I. es amplia, la cual ha sido estudiada por una serie importante de investigadores. Por ejemplo, para Mercader (2010) el valor intangible más importante es la credibilidad; para Muñoz (2010) son lealtad, conciencia y calidad; para López \& Sebastián (2009) son la ética, buen gobierno, innovación, calidad, responsabilidad y visión. A manera de resumen, se describen algunos de los investigadores que han relacionado los V.I. de las marcas (Tabla 1): 
Tabla 1: Valores Intangibles

\begin{tabular}{|c|c|}
\hline Autor & Valor Intangible \\
\hline Larkin (2003) & Trust \\
\hline Quevedo (2003) & Legitimación \\
\hline Davies, Chun, Vinhas \& Roper (2003) & Symmetry, affinity, connection \\
\hline Herranz (2003) & Excelencia, calidad, prestigio \\
\hline Alsop (2004) & Financial performance, quality of products, corporate leadership, vision \\
\hline Jackson (2004) & Quality, innovation, profitability, loyalty \\
\hline Fombrun \& Van Riel (2004) & Authentic, visible, transparent, consistent, distinctive \\
\hline Covey (2007) & Confianza \\
\hline Fundación Alternativas (2007) & $\begin{array}{l}\text { Confianza, transparencia, integridad, responsabilidad, integración, inclusión, } \\
\text { compromiso }\end{array}$ \\
\hline López \& Sebastián (2009) & Ética, buen gobierno, innovación, calidad, responsabilidad, visión \\
\hline Griffin (2009) & Ethics, sustainability, responsibility \\
\hline Pizzolante (2009) & Confianza, transparencia \\
\hline Molleda (2010) & Autenticidad, valores, tradición \\
\hline Mercader (2010) & Credibilidad \\
\hline Muñoz (2010) & Lealtad, conciencia, calidad \\
\hline Ewing (2011) & Philanthropy, strategy, expertise \\
\hline Komisarjevsky (2012) & Character, trust, communication \\
\hline Shepherd, Chartrand \& Fitzsimons (2015) & $\begin{array}{l}\text { Ideology, satisfaction, power, status, wealth, control, dominance, resourceful, } \\
\text { universalism, concern, equality, social justice, achievement, hedonism, stimulation, } \\
\text { self direction, benevolence, tradition, conformity, security. }\end{array}$ \\
\hline Schultz \& Block (2015) & Loyalty, sustainability, equity, profitability. \\
\hline Villagra, López \& Monfort (2015) & $\begin{array}{l}\text { Corporate governance, communication, social corporate responsibility (SCR), } \\
\text { legitimacy. }\end{array}$ \\
\hline Apolo, Báez, Pauker \& Pasquel (2017) & Corporate communication, commitment, knowledge, understanding, awareness. \\
\hline
\end{tabular}

Fuente: Elaboración propia

Los anteriores V.I. han sido descritos por diversos autores como aquellos que las marcas deben conservar dentro de su comunicación, con el fin de generar un mejor vínculo con cada uno de los stakeholders. Es el caso de las cervezas artesanales, que constituyen un tipo de producto que se ha decantado por la generación de valores intangibles como un recurso comunicativo para que las diferencien de marcas de producción masiva, evidenciado en proporciones de producción, tratamiento de la materia prima y procesos de elaboración. 


\subsection{La cerveza artesanal}

Las cervecerías artesanales se definen como pequeñas empresas productoras de cerveza, independientes y tradicionales, pues su operación no pasa de seis millones de barriles de cerveza anualmente, las cuales utilizan ingredientes no tradicionales con fermentaciones innovadoras (Fish, 2015). Para Reid \& Gatrell (2015) se presenta un crecimiento exponencial en el surgimiento de marcas de cerveza artesanal, ya que en Estados Unidos se ha pasado de 8 a 3400 cervecerías artesanales en tan solo 34 años. En 2012 esta industria contribuyó con 33.9 billones de dólares a la economía de Estados Unidos, y fue responsable por más de 360,000 empleos. En otro sentido, investigaciones realizadas por Fletchall (2016) advierten que aunque la producción per cápita de cerveza en los Estados Unidos ha disminuido desde los años 80, el número de cervecerías ha aumentado de 80 en 1983 a más de 4.000 en septiembre de 2015, de las cuales el 99\% de ellas corresponde a pequeños productores.

Roberson \& Collins (2015) ilustran el caso de Sudáfrica, y la relación entre aspectos culturales de la región que son fuertes motivadores para el turismo, y la visita y consumo de pequeñas cervecerías artesanales. Se evidencian casos parecidos en países como Reino Unido, Eslovaquia y Australia, donde se alberga festivales y eventos relacionados con la cerveza artesanal. Los autores resaltan las oportunidades del turismo por ser un motor de desarrollo local y regional sostenible, como una base de la economía local promoviendo la reafirmación de identidades y tradiciones locales. Šušterši \& Šušterši (2013) encuentran al respecto que en el mercado de la cerveza en Eslovaquia, hubo importaciones de cervezas industriales, que pensaron afectarían a las pequeñas industrias. Pero los consumidores prefirieron las artesanales pues percibían la importancia del sabor de la cerveza y la diversidad que les brindaban. Autores como Fletchall no sólo da importancia al producto como tal sino al lugar, afirmando que "Place comes into existence when meaningful experience is attached to a particular location" (2016: 539), demostrando cómo las cervecerías artesanales desempeñan un papel en la creación contemporánea de lugares, pues brindan la oportunidad de experimentar la comunidad local de una manera auténtica, y por lo tanto, ofrecer una oportunidad para hacer una conexión significativa con el lugar.

Ahora bien, en Colombia se venden 21 millones de hectolitros de cerveza y aunque las tres empresas insignia de cerveza artesanal, BBC Company (Bogotá), Tres Cordilleras y Apóstol (Medellín), apenas tienen una producción al año de alrededor de 36.000 hectolitros, lo que representa un crecimiento por encima del $30 \%$ al año (Revista Dinero, 2015). Dos de estas tres marcas de cerveza artesanal que vienen en crecimiento exponencial son producidas en la ciudad de Medellín, por lo que estudiar los intangibles de marcas de cerveza artesanal y cómo estos son construidos por sus consumidores es un tema relevante. Asimismo, este fenómeno ha venido tomando fuerza en los últimos años por el potencial económico de crecimiento y oportunidades para el desarrollo de economías locales, y por las construcciones sociales que generan estas marcas artesanales en relación con los consumidores.

Entender el impacto de estas marcas de cerveza artesanal en los consumidores aportaría al entendimiento de nuevas formas de consumo, desde cómo estas promueven su identidad y cómo se ve reflejada en la imagen que los consumidores crean a partir de la construcción de los valores intangibles que la marca les propone. Bajo esta perspectiva, esta investigación podría contribuir a entender mejor cómo se viene desarrollando este sector en la mente de los consumidores. Por 
lo tanto, este estudio pretende analizar el proceso de construcción de los V.I. que hace el consumidor de las marcas de cerveza artesanal producidas en Medellín, a fin de correlacionarse con la estrategia de comunicación que estas emiten.

\section{Metodología}

Esta investigación es cualitativa, con un enfoque hermenéutico-etnográfico y de alcance correlacional. Consistió en llevar a cabo 4 pasos consecutivos. El primero se enfocó en hacer un análisis del discurso de las páginas web de las compañías cerveceras Apóstol y 3 Cordilleras, marcas colombianas producidas en la ciudad de Medellín. El análisis del discurso se hizo a partir del modelo que propone Prieto (1999) que plantea la evaluación de aspectos objetivos del discurso relacionados con el tema principal, el texto, los elementos morfológicos y el modelo comunicativo; además de aspectos subjetivos compuestos por valores y estereotipos, recursos expresivos utilizados, estrategia comunicativo-persuasiva, relaciones verbal visuales, aportes de la estructura narrativa, funciones del texto y el público objetivo. Para este análisis se utilizó como instrumento una tabla en Excel que permitiera realizar la comparación de los aspectos anteriormente mencionados.

En segunda instancia, se realizaron entrevistas semiestructuradas a expertos, considerados de esta manera por ser las personas que lideraban la comunicación estratégica de las marcas investigadas. Previamente se realizó una prueba piloto de la herramienta con un experto en marcas importantes de cerveza colombiana, quien trabaja en el área de mercadeo como líder de la estrategia comunicativa. Basados en la prueba piloto, se realizaron cambios, dando como resultado una herramienta con 15 preguntas enfocadas a la estrategia comunicativo-persuasiva de la marca, los valores intangibles emitidos, la intención del mensaje y el enfoque en el consumidor. Finalmente dentro de esta parte del proceso, se entrevistaron 5 expertos, en donde se encontraban los community managers de ambas compañías, los dueños de las cervecerías, publicistas y directores de mercadeo.

El tercer paso consistió en realizar un análisis del discurso de los tours cerveceros. El trabajo de campo se realizó atendiendo a este tour, grabando el discurso del guía y analizando posteriormente la información bajo el mismo modelo y herramientas anteriormente mencionadas para las páginas web. Los tours son eventos que hacen cada jueves en las plantas de producción de ambas microcervecerías. En ellos, se hace un breve recorrido por la planta con el objetivo de informar sobre la empresa, los modelos de fabricación y la cerveza artesanal. Cuando ha terminado la charla, las personas se ubican en un pequeño bar dentro de la planta y degustan los diferentes tipos de cerveza producida.

Por último, se llevaron a cabo triadas de conversación con consumidores, basadas en el modelo de sesiones en profundidad o grupos de enfoque de Hernandez-Sampieri, Fernández-Collado \& Baptista-Lucio (2010), quienes sugieren que "el tamaño de los grupos varía dependiendo del tema: tres a cinco personas cuando se expresan emociones profundas o temas complejos" (2010: 426). Se realizaron en total 3 triadas de conversación, por lo que participaron 9 personas en total. Este ejercicio dejó de ejecutarse en el momento en que se llegó al principio de saturación de los datos. En ellas, se suscitaba a un debate con el objetivo de detectar en sus argumentos las construcciones relacionadas con la cerveza artesanal, la comunicación de las dos marcas y los valores intangibles detectados. Para este paso del trabajo de campo también se diseñó una guía de 15 preguntas semi estructuradas. 
Toda la información resultante de este trabajo de campo fue grabada y luego procesada por medio del software Atlas.ti para el análisis de los datos cualitativos y comprobación de categorías emergentes. La información primero fue catalogada en dos unidades hermenéuticas. En ellas los datos eran clasificados en códigos, posteriormente agrupados en familias de información y por último unidas en 4 grandes estructuras que resultaron siendo las categorías de análisis del estudio: estrategia comunicativa y valores intangibles de marca, consumidor y cerveza artesanal.

Estos resultados no necesariamente se evidenciarán mediante los cuatro pasos que se enuncian en la metodología, pues los datos fueron analizados de manera holística, basados en la interpretación de los valores intangibles desde los diferentes puntos de vista: mensajes emitidos por las marcas, percepción de los expertos y la construcción de valores intangibles que realizan los consumidores.

\section{Resultados}

\subsection{Los V.I. desde la estrategia comunicativa de las marcas. El análisis del discurso}

El análisis del discurso de las páginas web y de los tours cerveceros de ambas marcas fue un rastreo preliminar de las estrategias comunicativas y los V.I. que intentan inculcar en los consumidores. Dentro de los hallazgos más significativos se encuentra que ambas marcas persuaden al consumidor para que tenga un vínculo emocional desde las comunidades de marca y los tours cerveceros, como espacios en donde puede darse una culturización de la cerveza artesanal. Esto se entiende no como un proceso casero, sino como una fabricación cuidadosa desde pequeños lotes de producción y alta tecnología utilizada para el cuidado de los ingredientes, y en donde la cultura cervecera se enfoca en la experimentación de sabores, olores, aromas y composición del producto.

El objetivo principal de la marca Apóstol es comunicar la experticia en la fabricación de un producto premiumy artesanal. El mensaje es extenso y saturado pues se estructura en lo informativo de cuestiones como las recetas, tiempos de maduración, uso de ingredientes alemanes y belgas, los diferentes momentos de consumo y su maridaje; los jarros y botellas utilizadas para cada cerveza y las medallas y premios obtenidos. Esta marca enfoca su comunicación a V.I. como calidad, excelencia, tradición, prestigio y exclusividad.

Por su parte, 3 Cordilleras da prioridad a la comunicación de V.I. como diversidad, diversión y tranquilidad, acentuando su interés por el arraigo que desea inculcar en sus consumidores. Brinda importancia a ser una cervecería local que genera nuevas experiencias sensoriales desde lo colombiano, lo cual es símbolo de la pluralidad y variedad de modos de vida, fauna y flora, intentando transmitirlo a los consumidores por medio de sensaciones: mayores en sabor, color y aromas, inclusive desde el uso de ingredientes cultivados localmente. Los nombres de las cervezas dan cuenta de las diferentes razas del país. El discurso tiene una estructura simple pues la información que se entrega es clara y directa. Su foco son las experiencias fuera de la rutina, llenas de música, amigos y disfrute. 


\subsection{Los expertos}

Se nombraron como expertos a las personas que lideran la estrategia comunicativa de ambas marcas (community managers, publicistas, directores de mercadeo y accionistas). El objetivo de las entrevistas semiestructuradas consistió, a partir del alcance correlacional de la investigación, verificar la coherencia entre los mensajes de la marca y los valores intangibles emitidos en los tours cerveceros y sus páginas web.

Las entrevistas a expertos permiten encontrar que ambas marcas llevan a cabo estrategias BTL (below the line), marketing experiencial y herramientas de relaciones públicas, que se evidencian en la generación de arraigo y orgullo de la producción local. Lo artesanal, en el imaginario de los expertos es concebido desde la calidad, tanto por la materia prima como por la maquinaria y los tiempos de cocción. Se traduce en lotes pequeños elaborados cuidadosamente con ingredientes singulares. Lo anterior tiene el objetivo de transmitir al consumidor un concepto de sentirse único, exclusivo y diferente, al posicionar la cerveza artesanal como un nuevo bien de lujo exotizante. En ello, se vuelve importante la comunicación de la innovación en sabores, aromas y colores insignia de un consumo de lo experiencial que viene consigo, con una carga informativa como un manual de uso del producto llamado cultura cervecera.

Se habla de cultura cervecera, que es: disfrutar de buenos momentos, olores, sabores, colores de la cerveza, de una buena compañía y maridaje. Un buen servir, una buena temperatura. Que el consumidor no sea uno más del montón (Community Manager, Apóstol).

Los intangibles más importantes que ambas marcas desean transmitir y que no se encontraron anteriormente en el análisis del discurso son la cercanía, pues se facilita la conformación de comunidades de marca exclusivas; la irreverencia, como una actitud de la juventud con la cual puede haber un fenómeno de identificación con estas marcas artesanales, pues es un producto que respeta la esencia y tradición, lo que lleva a un último intangible en común que es la pasión por este tipo de fabricación al obtener resultados no industrializados, más orgánicos y naturales.

Esta cerveza es excelente. La base del trabajo es el producto. Una cerveza de excelente calidad, buenos procesos, buenas materias primas. Que no tiene nada que envidiarle a ninguna otra cerveza. Tratamos de transmitir que la marca es cercana, que tengan una interacción con nosotros de forma amistosa (Líder cervecero, 3 Cordilleras).

No obstante, cada una de las marcas tiene diferencias en el foco estratégico de su comunicación. En Apóstol es una prioridad la transmisión de V.I. como elegancia, tradición, premium, calidad y orgullo. Mientras que 3 cordilleras enfatiza en arraigo a la colombianidad, frescura, emocionalidad y extroversión. Si bien todos estos valores intangibles coinciden con aquellos comunicados en la página web y los tours, hay ligeras diferencias entre las opiniones de cada uno de los entrevistados, incluso dentro de la misma marca, lo cual permite dilucidar que unos expertos dan más peso a unos V.I. que otros.

\subsection{Los V.I. desde el consumidor}

Luego del análisis del discurso de las comunicaciones web y los tours cerveceros, las entrevistas con los expertos, se realizaron una serie de triadas, mediante una conversación con 3 consumidores de las marcas, con los cuales se incentivaba un debate a profundidad alrededor de los valores intangibles que perciben de Apóstol y de 3 Cordilleras. 
Dentro de los hallazgos más significativos se encuentra que hay dos tipos de consumidores de cerveza artesanal. Aquellos que son heavy users y tienen mayor información sobre este segmento, y consumidores eventuales que comienzan con un consumo más regular. El primer grupo presentó una preferencia por Apóstol, para la cual rescataron V.I. como excelencia, calidad, prestigio, distinción, tradición y experticia; mientras el segundo grupo, que prefería a 3 Cordilleras, encontraron relevantes otros como afinidad, lealtad, y compromiso (vinculados al amor por Colombia y la cercanía de la marca). Los consumidores de las triadas perciben a 3 Cordilleras como una marca que hace énfasis en el arraigo a lo colombiano, que genera sentimientos de frescura, relajamiento, tranquilidad y cercanía, convirtiéndose en una opción más emocional para el consumidor. Por otro lado, Apóstol sólo es entendida por los heavy users como una marca con mejor sabor y elaboración de la cerveza, dando prioridad a la composición y calidad del producto, la tradición cervecera y la experticia en su fabricación. Los consumidores eventuales ven a Apóstol como una marca que aunque hace sentir un orgullo por la calidad de un producto colombiano, igual de bueno al extranjero, no genera la afinidad que si produce 3 Cordilleras. Por otro lado, en todos los consumidores se encuentran consensos en cuanto al concepto de lo artesanal hacia ambas marcas. Entre ellos están los V.I. como confianza, conexión, autenticidad, innovación y diversidad. Estos se relacionan a un concepto en común, que si bien es emitido de forma diferenciada por las estrategias de ambas marcas, hace referencia a una construcción simbólica que el consumidor ha hecho de lo artesanal, concebido como una forma de volver a lo tradicional, a lo casero.

Lo artesanal es que alguien se tomó el trabajo de amasar, de mezclar los ingredientes como la receta de la abuela. Uno siente que lo artesanal es un privilegio, algo de pocos, que se hizo con mucho cuidado. Es como el día que la abuela cocinó algo exquisito y precisamente ese día le quedó particularmente bueno, y uno no sabe si eso se repite. Es el privilegio de haber estado allí (Hombre, 28 años).

Se busca en la cerveza artesanal lo diferente, vibrante, raro, e incluso extravagante. El consumidor, encuentra en este producto una búsqueda de identidades nuevas desde lo propio, entendido como el orgullo de lo colombiano. En lo artesanal hay un encuentro con un nuevo lujo, en donde al ser una forma exclusiva y diferente de tomar cerveza, se generan rituales que acompañan el consumo, evidenciado en los tours cerveceros, en donde el conocimiento de los ingredientes, el proceso, la planta de producción y los vasos especiales así lo confirman.

El consumo de la cerveza artesanal es una necesidad por redefinirse...como el sushi, búsquedas de identidades nuevas...pero desde lo propio, es un ánimo de despertarse, disfrutar más (Hombre, 30 años).

Basado en lo artesanal, el consumidor se encuentra con una sensación de privilegio, en donde no existe problema en que no tenga los mismos estándares de calidad de una cerveza industrial, pues por el contrario, entre más casero, cuidadoso y manual sea, se percibe como mejor por el empeño y el trabajo puesto en su elaboración. Por ello, el consumidor de cerveza artesanal se entiende como un sujeto que se concibe a sí mismo como joven de espíritu, abierto y atrevido. Tiene la necesidad de sentir a la marca como propia, es decir, de pertenencia e identificación.

Finalmente, y luego del desarrollo del análisis del discurso, las entrevistas semiestructuradas y las triadas, se pueden encontrar una serie de valores intangibles que pueden ser comparados desde las estrategias de comunicación de las marcas, de las concepciones de los expertos y desde las percepciones de sus consumidores finales (Tabla 2). 
Tabla 2. Los V.I. desde la emisión hasta la percepción del mensaje

\begin{tabular}{|c|c|c|c|}
\hline \multicolumn{3}{|c|}{ Estrategia comunicativa de la marca } & \multirow{2}{*}{$\begin{array}{c}\text { Consumidor } \\
\text { Triadas de conversación }\end{array}$} \\
\hline & Análisis del discurso & Entrevista a expertos & \\
\hline Apóstol & $\begin{array}{l}\text { calidad } \\
\text { excelencia } \\
\text { tradición } \\
\text { prestigio } \\
\text { exclusividad }\end{array}$ & $\begin{array}{c}\text { calidad } \\
\text { elegancia } \\
\text { tradición } \\
\text { lo premium (prestigio) } \\
\text { orgullo }\end{array}$ & $\begin{array}{l}\text { calidad } \\
\text { excelencia } \\
\text { tradición } \\
\text { prestigio } \\
\text { distinción } \\
\text { experticia }\end{array}$ \\
\hline 3 cordilleras & $\begin{array}{l}\text { diversidad } \\
\text { diversión } \\
\text { tranquilidad } \\
\text { arraigo }\end{array}$ & $\begin{array}{l}\text { emocionalidad } \\
\text { extroversión } \\
\text { frescura } \\
\text { arraigo }\end{array}$ & $\begin{array}{c}\text { afinidad } \\
\text { lealtad } \\
\text { compromiso } \\
\text { comunicación } \\
\text { cercanía }\end{array}$ \\
\hline Ambas marcas & $\begin{array}{l}\text { emocionalidad } \\
\text { experiencialidad } \\
\text { innovación } \\
\text { autenticidad }\end{array}$ & $\begin{array}{c}\text { orgullo } \\
\text { calidad } \\
\text { innovación } \\
\text { cercanía } \\
\text { irreverencia } \\
\text { pasión } \\
\text { Autenticidad }\end{array}$ & $\begin{array}{c}\text { confianza } \\
\text { conexión } \\
\text { autenticidad } \\
\text { innovación diversidad }\end{array}$ \\
\hline
\end{tabular}

Fuente: Elaboración propia

\section{Conclusiones}

Dentro de las conclusiones más significativas del estudio se encuentra que las cervezas artesanales se están desarrollando como un nuevo tipo de segmento, como resultado de la mediación de nuevas narrativas y estrategias comunicativas por parte de las marcas, que enfocan la comunicación a la exclusividad, autenticidad, calidad y cuidado en la fabricación. De esta forma han convertido a la cerveza en un producto propenso a experimentos gustativos, que permiten nuevos momentos de consumo, acompañados de rituales que incluyen formas de encuentro como los tours cerveceros y festivales; además de experiencias sensoriales que incluyen sabores, aromas y colores, resultado del uso de ingredientes innovadores. Esto en coherencia con las afirmaciones de Fish (2015), quien sostiene que el centro del negocio de las cervecerías artesanales es el uso innovador, tanto de ingredientes y fermentaciones, como en sus técnicas de la elaboración.

En el discurso de ambas cervecerías se encuentra la búsqueda de producciones locales y pequeñas, con el fin de darle protagonismo al producto, su sabor y calidad, por encima de la producción masiva y también a la construcción de lu- 
gar, lo cual permite la apropiación de constructos socio-culturales de identidad colombiana. En esta concepción de la fabricación, se alberga, además, un imaginario de lo artesanal, que intenta transmitir al consumidor valores intangibles como la exclusividad, diversidad y calidad. Sin embargo, dentro de las construcciones que hacen los consumidores de esta noción, se encuentran grandes diferencias respecto a las generadas por las marcas, pues al no ser las peculiaridades de la fabricación y el uso de tecnología de punta la prioridad, sí lo es el retorno a lo esencial, lo natural y orgánico, nombrado como lo casero, que también hace alusión a apropiarse de lo local y lo colombiano. En esta elaboración se puede dilucidar un fenómeno social y cultural relacionado al rechazo de lo industrial como símbolo de lo masificador, común y estructuralmente imbatible. La cerveza artesanal, permite entonces al consumidor tangibilizar en un producto la necesidad de sentirse diferente e identificarse con lo exótico, como un intento por descubrir lo propio. En ese mismo sentido, los V.I. como el arraigo y el orgullo detectados en la intención comunicativa de ambas marcas, satisfacen la búsqueda de los consumidores de identidades nuevas a partir de lo propio. Esto coincide con las afirmaciones de Roberson \& Collins (2015) quienes sostienen que existe una fuerte relación entre la cerveza artesanal y las oportunidades que genera el promover la reafirmación de identidades y tradiciones locales. Fletchall (2016) también demuestra lo anterior en cuanto a que las cervecerías artesanales efectivamente cumplen con una función en la creación contemporánea de lugares, pues por medio de lo experiencial, la comunidad local puede sentirse identificada con lo auténtico, y por lo tanto, ofrecer una mayor conexión con la marca.

Esta investigación permite demostrar que el concepto de lo artesanal es un condicionante de la forma en cómo tanto las marcas como el consumidor construyen los valores intangibles. Por parte de 3 Cordilleras y Apóstol la identidad de marca es construida desde V.I. como la calidad y la innovación, por la fabricación cuidadosa de pequeños lotes de producción pero con el uso de plantas modernas. Por otro lado, la imagen de marca que han construido los consumidores de ellas ha sido más enfocada a la confianza, conexión, autenticidad y diversidad. Estos V.I. se ven más orientados a la necesidad de la cercanía de la marca con los consumidores al buscar una opción diferenciadora e irreverente en donde se prioriza la esencia sobre la cantidad. Dentro de aquellos valores intangibles en los que se encuentra una correlación tanto desde la estrategia comunicativa hasta la percepción del mensaje, están la experiencialidad y autenticidad para ambas marcas. Apóstol particularmente, logra la transmisión y percepción exitosa de V.I. como la calidad, tradición y prestigio. Este último puede encontrar sinónimos en sus consumidores como elegancia, lo premium y la distinción. No obstante, esta marca no logra comunicar satisfactoriamente que es colombiana, ya que solo un pequeño porcentaje de los consumidores lo entiende. Por su parte, 3 Cordilleras sí logra transmitir el arraigo a lo colombiano, pues los consumidores asumen V.I. como afinidad, lealtad y compromiso.

Este estudio muestra la importancia de investigar los valores intangibles, construidos tanto desde la identidad como desde la imagen, pues evidencia el poder de las comunicaciones para la construcción de nociones y concepciones de marca, y la apertura de nuevos segmentos de consumo mediados por dichas estrategias comunicativas. La investigación inclusive pone en evidencia cómo estas nociones construidas tanto desde la marca como desde el consumidor pueden afectar la manera en cómo se erigen desde los imaginarios y lo simbólico los V.I. de marca.

Sería importante para futuras investigaciones, examinar otros canales comunicativos utilizados por estas micro cervecerías, como festivales de cerveza artesanal y eventos especiales en donde llevan a cabo sus estrategias de comunicación 
y mercadeo. Por otro lado, sería interesante profundizar respecto al fenómeno de lo artesanal en otros sectores empresariales, de tal forma que se puede evidenciar cómo se comporta esta creciente tendencia respecto a otras estrategias comunicativas y a los V.I. que emergen en los consumidores.

\section{Referencias bibliográficas}

Alsop, R. (2004). The 18 immutable laws of corporate reputation. Creating, protecting \& repairing your most valuable asset. London: Kogan Page.

Apolo, D., Báez, V., Pauker, L. \& Pasquel, G. (2017) Corporate Communication Management: Considerations for the approach to its study and practice. Revista Latina de Comunicación Social, 72, 521 to 539.

Buil, I., Catalán, S. \& Martínez, E. (2016). The importance of corporate brand identity in business management: An application to the UK banking sector. Business Research Quarterly, 19 (1), 3-12.

Che-Hui, L., Miin-Jye, W., Li-Ching, H. \& Kuo-Lung, W. (2015). Online hotel booking: The effects of brand image, price, trust and value on purchase intentions. Asia Pacific Management Review, 20, 210-218.

Chao-Sen W. (2015). A study on consumers' attitude towards brand image, athletes' endorsement, and purchase intention. The International Journal of Organizational Innovation, 8 (2), 233-253.

Covey, S. (2007). El factor confianza. El valor que lo cambia todo. Barcelona: Paidós.

Davies, G., Chun, R., Vinhas, R. \& Roper, S. (2003). Corporate reputation and competitiveness. New York: Routledge.

Elizondo, D.E, Bouchard, C. \& Favart, C. (2016). Defining Brand Identity Through Kansei-Experience Approach, the Creation of the Experience Framework Boards (EFB). Journal of Integrated Design \& Process Science. 20 (2), 101-112.

Ewing, A. (2011). Corporate responsibility. En Doorley, J. \& García, H. (Eds.), Reputation Management. The key to successful public relations and corporate communication (353-381). New York: Routledge.

Finne, A \& Grönroos, C. (2009). "Rethinking marketing communication: From integrated marketing communication to relationship communication", Journal of marketing Communications, 15, 179-195.

Fish, H. (2015). Effects of the Craft Beer Boom in Virginia: How Breweries, Regulators, and the public can collaborate to mitigate environmental impacts. William \& Mary Environmental Law \& Policy Review, 40 (1), 273-304. 32

Fletchall, A. (2016). Place-making throurgh beer-drinking: A case study of Montana's Craft breweries. Geographical Review 106 (4), 539-566.

Fombrun, C. \& Van Riel, C. (2004). Fame \& Fortune. How successful companies build winning reputations. New Jersey: Prentice Hall.

Fundación Alternativas. (2008). La confianza social en las empresas españolas. Madrid: Fundación Alternativas.

Gázquez, J. \& Sánchez, M. (2004). La identidad e imagen de marca. En Jiménez, A. (Ed.), Dirección de productos y marcas (56-82). Barcelona: UOC. 
Gehani, R. (2016). “Corporate Brand Value Shifting from Identity to Innovation Capability: from Coca-Cola to Apple”. Journal of Technology Management \& Innovation. 11(3), 11-20.

Griffin, A. (2009). New strategies for reputation management. Gaining control of issues, crises and corporate social responsibility. London: Kogan Page.

Hernandez-Sampieri, R., Fernández-Collado, C. \& Baptista-Lucio, M. (2010). Metodología de la Investigación. México: McGraw Hill.

Herranz, J. (2004). La comunicación que fortalece la imagen y la reputación corporativa. En Herranz, J. \& Salinas, F. (Eds.), La comunicación en el ámbito de la economía social (19-47). Ávila: Universidad Católica de Ávila.

Jackson, K. (2004). Building reputational capital. Strategies for integrity and fair play that improve the bottom line. New York: Oxford University Press.

Kapferer, J. (1992). La marca, capital de la empresa. Bilbao: Deusto.

Keller, K. (2008). Administración estratégica de marca branding. México: Pearson.

Keller, K. (2016). Unlocking the Power of Integrated Marketing Communications: How Integrated Is Your IMC Program?, Journal of Advertising, 45 (3), 286-301.

Komisarjevsky, C. (2012). The power of reputation. Strengthen the asset that will make or break your career. New York: Amacom.

Larkin, J. (2003). Strategic reputation risk management. New York: Palgrave. MacMillan.

López, B. \& Sebastián, A. (2009). Responsabilidad social corporativa y reputación corporativa. En Sánchez, J. \& Pintado, T. (Eds.), Imagen corporativa. Influencia en la gestión empresarial (139-170). Madrid: ESIC.

Manhas, P., Manrai, L. \& Manrai, A. (2016). Role of tourist destination development in building its brand image: A conceptual model. Journal of Economics, Finance \& Administrative Science, 21(40), 25-29.

Mercader, J. (2010). Fundaciones laborales, herramienta para canalizar la responsabilidad social empresarial. Valencia: Tirant lo Blanch.

Millares-Marcelo, J., Millares-Quirós, M. \& Daza-Izquierdo, J. (2014). “Reputación Corporativa y creación de valor para el accionista". Business Review, 44, 16-33.

Molleda, J. (2010). Identidad, autenticidad y reputación: una triada dinámica. En Sólanich, F. (Ed.), Relaciones públicas: reflexiones y desafios (22-31). Santiago de Chile: Universidad del Pacífico.

Muñoz, M. (2010). Reputación corporativa: trustmark y activo de comportamientos adquisitivos futuros. Cuadernos del Centro de Estudios en Diseño y Comunicación, 33, 23-40.

Orozco-Toro, J. (2018). Reputación corporativa. Gestión y comunicación de los valores intangibles de marca. Medellín: UPB. 
Pizzolante, I. (2009). Asumir el desafío de modelar el nuevo entorno empresarial o adecuarse a él. En Costa, J. (Ed.), Dircom. Estratega de la complejidad. Nuevos paradigmas para la dirección de la comunicación (77-86). Barcelona: Aldea Global.

Porcu, L., Del Barrio-García, S. \& Kitchen, P. (2012). How Integrated Marketing Communications (IMC) works? A theoretical review and an analysis of its main drivers and effects, Comunicación y Sociedad, 15 (1), 313-348.

Prieto, D. (1999). El juego discursivo: manual de análisis de estrategias discursivas. Buenos Aires: Lumen Humánitas.

Quevedo, E. (2003). Reputación y creación de valor. Madrid: Thomson.

Reid, N. \& Gatrell, J. (2015). Brewing Growth. Economic Development Journal, 14, 5-12.

Revista Dinero (8 de Julio de 2015). ¿Cuál ha sido el secreto del éxito de las cervezas artesanales? http://www.dinero.com/ edicion-impresa/negocios/articulo/cual-ha-sido-secreto-del-exito-cervezas-artesanales-colombia/212031

Roberson, C. \& Collins, K. (2015). Beer Tourism in South Africa: Emergence and Contemporary Directions. Nordic Journal of African Studies, 24, 241-258.

Sanz de la Tajada, L. (1996). Auditoría de la imagen de empresa. Métodos y técnicas de estudio de la imagen. Madrid: Síntesis. Schultz, D. \& Block, M. (2015). Beyond brand Loyalty: Brand sustainability. Journal of Marketing Communications, 21, 340-355.

Semprini, A. (1995). El marketing de la marca. Una aproximación semiótica. Barcelona: Paidós.

Shepherd, S., Chartrand, T. \& Fitzsimons, G. (2015). When Brands Reflect Our Ideal World: The Values and Brand Preferences of Consumers Who Support versus Reject Society's Dominant Ideology. Journal of Consumer Research, 42.

Slade, C. (2016). Creating a Brand Identity: A Guide for Designers. London: Laurence King Publishing.

Šušterši, J. \& Šušterši, S. (2013). Do Consumer Tastes Evolve with Competition? The Case of the Slovenian Beer Market, Kyklos, 66 (2), 306-316.

Turan, H. (2017). A research on relationship between brand image perceiving and customer loyalty levels in marketing management. Journal of International Social Research. 10 (48), 653-658.

Villagra, N., López, B. \& Monfort, A. (2015). The management of intangibles and corporate branding: Has anything changed in the relationship between business and society?. Revista Latina de Comunicación Social, 70, 793-812. 